\title{
Role of antioxidants in prevention of diseases
}

\begin{abstract}
Antioxidants are first line defence system against free radical damage and play very crucial role in maintaining optimum health care. Although, present day research is focused on the potential and miraculous benefits of antioxidant nutrients or supplements in our daily life. It has become the best protection against oxidative stress involve a wide selection of interrelated antioxidants and antioxidant cofactors. In addition, the combination of several suboptimal concentrations of these kinds of detoxifying supplements may also have an additive or even synergistic role to decrease the risk of some of common and lethal diseases which caused due to aging. Adequate intake of antioxidants, such as beta-carotene and vitamin C supplements or some of fruit and vegetables which have been reported as essential antioxidants which play a vital role in decreasing the risk of cancer and coronary heart disease (CHD).
\end{abstract}

Keywords: antioxidants, vitamin E, beta-carotene, vitamin C, lipoic acid, treatment, inflammatory diseases, neurodegenerative diseases, cardiovascular diseases
Volume 4 Issue I - 2017

\author{
Kirti Rani \\ Amity University Uttar Pradesh, India
}

Correspondence: Kirti Rani,Assistant Professor (III), Amity Institute of Biotechnology, Amity University Uttar Pradesh, Noida, Sec-125, Gautam Buddha Nagar, Noida-201313 (UP), India, Tel +9|-|20-439-294-6, +9|-999-032-949-2,

Email krsharma@amity.edu, Kirtisharma2k@rediffmail.com

Received: April 26, 2017| Published: September 28, 2017

\section{Introduction}

Free radicals are responsible for causing pathogenesis of healthy cells to lose their structures and functions to develop various degenerative diseases that caused due to aging such as cancer, cardiovascular disease, cataracts, immune system decline, brain dysfunction as well as illness caused due to pollution, cigarette smoke, drugs, illness, stress. So, many dieticians believe that the Recommended Dietary Allowance (RDA) for specific antioxidants may be required to live healthy lifestyle with having well-balanced, wholesome diet and adequate antioxidant supplementation. A balanced diet including plenty of fruit, vegetables, grains, oils and nuts have excellent complement of antioxidants such as vitamin E, beta-carotene, vitamin $\mathrm{C}$ and lipoic acid which have their protective effect by decreasing oxidative damage to DNA and by Although, antioxidants are generally regarded as safe compounds, but even at high concentrations, they may generated reactive species which can have a pro-oxidant effect. ${ }^{1}$ Antioxidant enzymes also have an important role in the detoxification of free radicals such as superoxide dismutase, catalyse, and glutathione peroxidase which scavenge free radicals. Decrease in antioxidant defence system or an overproduction of free radicals results in oxidative stress was found to contribute many aging induced diseases. Therapies based on both antioxidants and antioxidant enzymes can be an effective approach in preventing or treating many diseases.

\section{Antioxidants in cancer prevention}

Carcinogenesis starts when a normal cell is transformed into a cancerous cell. A number of phytochemicals such as genistein, tea polyphenols, the soy isoflavone that are present in edible plants, have anticarcinogenic and antimutagenic effects and can interfere with a particular stage in the development of cancer for lowering the risk of developing some cancers particularly of the digestive and respiratory tracts. ${ }^{2}$ Grape seed proanthocyanidin extract (GSPE) has been shown to have a positive effect against free radicals, oxidative stress and tissue injury. ${ }^{3}$ The ability of lycopene to scavenge free radicals that can we considered to prevent oxidative damage to DNA, which partly explains its anticarcinogenic activity. ${ }^{4}$ Omega-3 fatty acids can also helpful in reducing inflammatory mediators and leukotrienes by producing superoxide anions. ${ }^{5}$

\section{Antioxidants in inflammatory diseases}

In rheumatoid arthritis, toxic substances are released from the synovium that cause inflammation of the joint tissues lead to the destruction of cartilage. Antioxidant based treatments may be better therapeutic approach for treating this disease such as introduce the French diet in daily meal. ${ }^{6}$ Vitamin E-alpha tocopherol, vitamin A and Vitamin B scavenge free radicals to react with lipids and proteins that considered a mild anti-inflammatory effect which consequently protecting lipids in cell membranes from peroxidation. ${ }^{7}$ Pycnogenol (Procyanidine B3) have a role in preventing many inflammatory diseases and it increases the intracellular synthesis of antioxidant enzymes which scavenges free radicals to reduce the production of peroxides by macrophages to protect the cells and tissues from oxidative stress. ${ }^{8}$ Lipoic acid is a naturally occurring antioxidant, which has been used clinically for the treatment of oxidant-induced diseases that scavenges free radicals that accelerates glutathione synthesis and modulates the activity of transportation factors such as nuclear factor-kB (NF-kB). ${ }^{9}$ Green tea, of tea plant, Camellia sinensis, contains polyphenolic compounds have been shown to reduce the inflammation associated with arthritis in mice which contain compounds called, catechins that inhibit the breakdown of proteoglycan and collagen. ${ }^{10}$

\section{Antioxidants in diabetes mellitus management}

Diabetes mellitus is a metabolic disorder, in which the beta cells in the pancreas is not release enough insulin lead to hyperglycaemia. The isoflavones genistein and daidzein, are the most active, and are mainly found in soybean and its products that derived from biochanin A and for mononetin which can helpful in diabetes management. As well as, dietary phytoestrogens, including isoflavones and lignans have a beneficial role in both obesity and diabetes due to their positive regulatory actions on glucose and lipid metabolism. Flaxseed has the highest concentration of lignans and they are also found in seeds, whole grains, legumes and vegetables. This lignans was found to have vital role in diabetes management as antioxidant. ${ }^{11}$

\section{Antioxidants in neurodegenerative disorders}

An increased production of reactive oxygen and nitrogen species has been shown to contribute to several neurodegenerative disorders 
that can be prevented by antioxidants intake. Improved memory is reported with the higher levels of carotenoid and tocopherol in individuals, whereas low levels of antioxidants are associated with a greater risk of brain vascular disease, memory loss, and dementia. Resveratrol predominantly found in grapes and red wine may also have a protective effect against Alzheimer's disease. ${ }^{12}$ Ascorbate can scavenge reactive species and superoxide anion, peroxyl, hydroxyl and peroxynitrite radicals, and lipid hydroperoxides that make catecholamines more stable, preventing them from producing reactive oxygen species. ${ }^{13}$

\section{Antioxidants toxicity}

Antioxidants toxicity especially of three antioxidants, vitamin E, beta-carotene and lipoic acid have been widely studied and evaluated for optimum health care when administrated in patients in the form of intravenous injections or taken orally. At high concentrations of vitamin $\mathrm{A}$, vitamin $\mathrm{C}$ and vitamin $\mathrm{E}$ can have an undesirable prooxidant effect that lead to increased chance of increase in fatal myocardial infarctions. Vitamin E can be metabolised to form quinone derivatives which are toxic to cells and produce oxygen radicals. Betacarotene is the precursor of vitamin A whose supplementation with beta-carotene increased cancer incidence in smokers and lipoic acid is made from fatty acids and the its R-enantiomer have antioxidant activity, but in the reduced form, dihydrolipoic acid, can also found to demonstrate a pro-oxidant effect. ${ }^{14}$

\section{Conclusion}

So, the review article contributes the useful and instant awareness for depicting the oxidative stress and free radicals induced diseases and their management by introducing clinical implementations of naturally occurring antioxidants or antioxidants supplements to prevent onset of many neurodegenerative disorders and cancers. The antioxidants have a strong potential to be used as most conventional treatments of diseases especially in, inflammatory disease, neurodegenerative diseases, cancer and diabetes. Hence, administration of balanced diet with good supplementation of fruit, vegetables, grains, oils and nuts) that have adequate essential antioxidant such as vitamin A, E, C, lipoic acid etc can be sufficient to improve our body immune system to prevent many neurodegenerative disorders, cardiovascular disease, premature aging, however, genetic and environmental factors may increase the risk of these kind of diseases at any stage.

\section{Acknowledgements}

I would like also to express my cordially appreciation to Amity University Uttar Pradesh, Noida (INDIA).

\section{Conflict of interest}

The author declares no conflict of interest.

\section{References}

1. Robert A Jacob. The integrated antioxidant system. Nutrition Research. 1995;15(5):755-766

2. Surh YJ. Cancer chemoprevention with dietary phytochemicals. Nat Rev Cancer. 2003;3(10):768-780.

3. Bagchi D, Bagchi M, Stohs SJ, et al. Free radicals and grape seed proanthocyanidin extract: importance in human health and disease prevention. Toxicology. 2000;148(2-3):187-197.

4. Ferrari CK, Torres EA. Biochemical pharmacology of functional foods and prevention of chronic diseases of ageing. Biomed Pharmacother. 2003;57(5-6):251-260.

5. Neil D Westcott, Alister D Muir. Flax seed lignan in disease prevention and health promotion. Phytochemistry Reviews. 2003;2(3):401-417.

6. Yannis Alamanos, Paraskevi V Voulgari, et al. Rheumatoid arthritis in Southern Europe: epidemiological, clinical, radiological and genetic considerations. Current Rheumatology Reviews. 2005;1(1):33-36.

7. McAlindon T, Felson DT. Nutrition risk factors for osteoarthritis. Ann Rheum Dis. 1997;56(7):397-400.

8. Rohdewald P. A review of the French maritime pine bark extract (Pycnogenol $\left.{ }^{\circledR}\right)$, a herbal medication with a diverse clinical pharmacology. Int J Clin Pharmacol Ther. 2002;40(4):158-168.

9. Packer L. Alpha-Lipoic acid: a metabolic antioxidant which regulates NF-kappa B signal transduction and protects against oxidative injury. Drug Metab Rev. 1998;30(2):245-275.

10. Adcocks C, Collin P, Buttle DJ. Catechins from Green tea (Camellia sinensis) inhibit bovine and human cartilage proteoglycan and Type II collagen degradation in vitro. J Nutr. 2002;132(3):341-346.

11. Bhathena SJ, Velasquez MT. Beneficial role of dietary phytoestrogens in obesity and diabetes. Am J Clin Nutr. 2002;76(6):1191-1201.

12. Marambaud P, Zhao H, Davies P. Resveratrol promotes clearance of Alzheimer's disease Amyloid- $\beta$ peptides. J Biol Chem . 2005;280(45):3737737382 .

13. Rice ME. Ascorbate regulation and its neuroprotective role in the rat brain. Trends Neurosci. 2000;23(5):209-216.

14. Bast A, Haenen GR. Interplay between lipoic acid and glutathione in the protection against microsomal lipid peroxidation. Biochim Biophys Acta. 1998;963(3):558-561. 